Article

\title{
Analysis of measurement conditions influence on the magnetic Barkhausen noise phenomenon
}

\author{
Michał Maciusowicz ${ }^{1 *}$, Grzegorz Psuj ${ }^{1}$ \\ 1 The West Pomeranian University of Technology in Szczecin, Poland \\ Prof. Grzegorz Psuj, gpsuj@zut.edu.pl \\ * Correspondence: Michał Maciusowicz M.Sc.; michal.maciusowicz@zut.edu.pl
}

Received: 05.07.2019; Accepted: 12.11.2019

\begin{abstract}
The effectiveness of the magnetic Barkhausen noise method (MBN), used for non-destructive testing of ferromagnetic materials, depends to a large extent on a number of factors determining the measurement conditions. The use of conditions allowing the highest possible level of discrimination between the various states of the materials state is of highest importance. Therefore, this paper presents an analysis of the impact of measurement conditions on Barkhausen noise signals observed for various states of the material conditions. Taking into consideration the stochastic nature of MBN and the complex characterization of its changes, the analysis was based on the time-frequency representation of the MBN signal. The paper presents selected distributions achieved using two transformation methods. In addition, the extraction methods of features allowing the quantification of complex information were given. Finally, the discrimination ability for a number of parameters and features of MBN signals were determined and the obtained results were discussed.
\end{abstract}

Keywords: magnetic Barkhausen noise; measurement conditions; time-frequency analysis; spectrogram; scalogram

\section{Introduction}

The importance of non-destructive inspection of the condition and properties of materials in modern industry is constantly growing. Many methods support both the processes of optimizing the properties of manufactured products and the subsequent assessment of their quality. One of the methods dynamically developed as part of the study of magnetic materials is the Barkhausen MBN (Magnetic Barkhausen Noise)

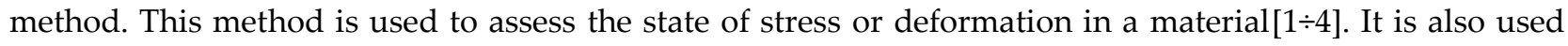
to study the microstructure properties of a magnetic material [1,5-7]. Its effectiveness depends not only on the tested material and its properties, but also on a number of measurement parameters, such as frequency, amplitude and shape of the field excitation current of the sample $[2,3,5,7 \div 14]$. Therefore, the key to the process of achieving high effectiveness of the assessment is the use of conditions that ensure the highest possible discrimination between the different states of the magnetic materials tested.

Most often, for the purposes of quantitative description of observed Barkhausen noise clusters, an analysis of its properties in the time domain and in the frequency domain is performed. The work [10] presents the results of studies on the impact of measurement conditions on MBN signals expressed in time or frequency domain. However, due to the stochastic nature of the measured MBN signals, analysis procedures have been developed using the combined representation of time and frequency, which allows obtaining holistic information about changes in the material [15:18]. This article uses two transformations to obtain time-frequency representations of MBN signals defining characteristics in time-frequency (TF) and time-scale (TS) space [19]. First, tests were carried out for a set of magnetic samples subjected to mechanical processes and representing different states of material properties, and, as a consequence, different magnetic properties. In the next stage, selected distributions were presented and an analysis of the impact of measurement conditions on the obtained discrimination was carried out using a number of proposed features of transformed signals. Finally, the obtained results are discussed. 


\section{Methodology of the experiment carried out}

The measurement experiment was carried out using four samples made of St3S structural steel. Before starting the measurements, three samples were loaded with static force of different characteristics, which resulted in obtaining various changes in the material structure. Sample s3 was subjected to a force in the material elasticity range. In contrast, samples s4 and s5 were subjected to forces exceeding the yield strength of the material, while in the case of sample s4 the value of the force in the loading process increased gradually, and in the case of s5 rapidly. Sample so was unloaded and is a reference sample. Changes in the material's mechanical structure cause changes in its magnetic properties. That is why the samples prepared in this way enabled testing of state discrimination in different measuring conditions.

The schematic diagram of the measuring system is shown in figure 1 . The prepared samples were checked with a Barkhausen magnetic noise measuring transducer. This transducer consists of two components: a magnetizing system and a system collecting the response field. These elements have been described in detail in publication [20]. The work of the entire system was managed using a computer (PC) equipped with a data acquisition card (DAQ) NI 6251 National Instruments containing analog and digital inputs and outputs. The voltage supply to the excitation coil was generated using the DAQ C/A module. Subsequently, the signal after amplification in the power amplifier was fed to the input of the transducer magnetizing system. In contrast, the response voltages that were collected using a measuring coil were first fed to the input of an analog signal processing system (ASP). This system allows bandwidth filtration $(1 \div 100 \mathrm{kHz})$ and amplification of the observed Barkhausen noise signal [20]. After passing through the appropriate blocks of the treatment process, this signal was saved on the computer disk. Next, digital procedures for processing and quantification of measurement signals were used.

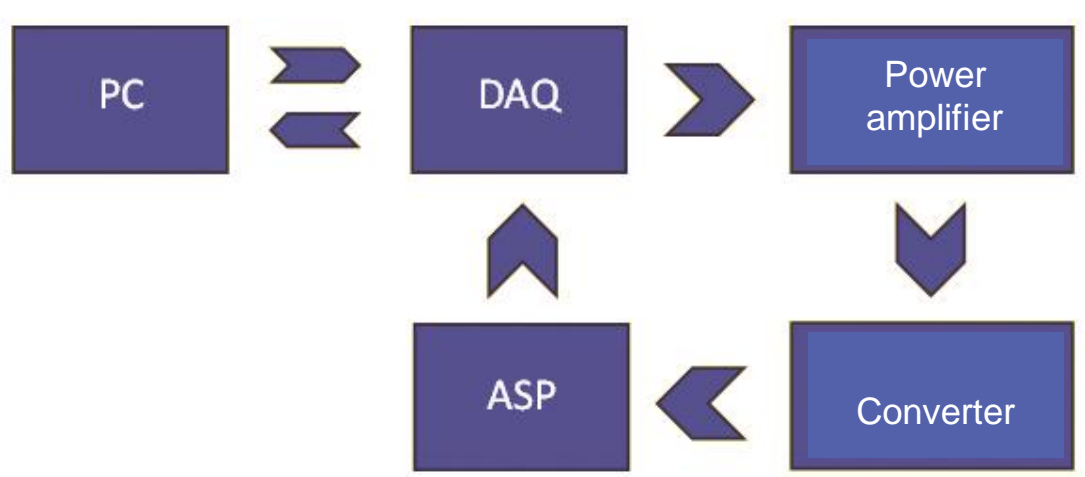

Fig. 1. Diagram of the measurement system; PC - control and measurement computer, DAQ - data acquisition card, ASP - analogue signal processing unit

The tests were carried out for various settings of the frequency and current shape of the magnetizing section. The frequencies used were: 0.5, 1, 2, 5, 10, 15, 20, 25 and $30 \mathrm{~Hz}$. During the measurements, sinusoidal and triangular current was used.

\section{Results and discussion}

After carrying out a series of measurements for the range of measurement parameters described in the previous section, an analysis of MBN signals was performed. It was divided into several parts. The first compares the qualitative representation of the MBN time-frequency signals resulting from the use of two types of transformations operating in the TS and TF space. In the second part, a quantitative analysis was carried out to obtain the features enabling the quantification of information carried by distributions. This allowed for a measurable assessment of the impact of various measurement conditions on obtaining the greatest discrimination of the states of the tested material.

First, the wavelet transform and Short-time Fourier Transform (STFT) were used to calculate the timefrequency distributions. As a result of the wavelet transformation, spatial distributions called scalograms were obtained. However, in the case of using the STFT transformation, spectral density distributions were obtained, called spectrograms [19]. Examples of distribution results obtained for the s3 sample using sinusoidal excitation are shown in figures 2 and 3. 

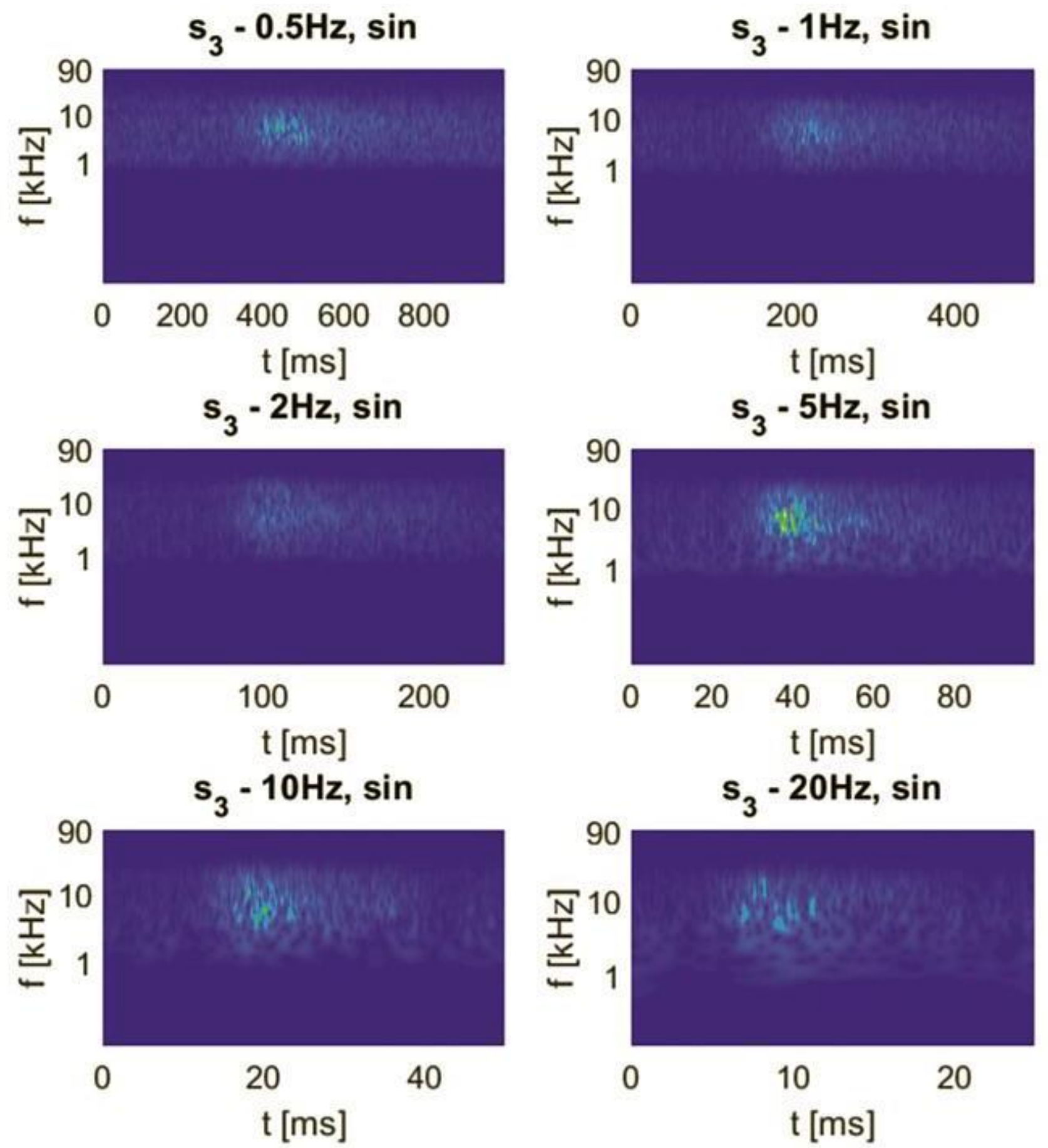

Fig. 2. Scalograms obtained for sample sз based on MBN signals acquired for different excitation frequency values

The scales presented in figure 2 illustrate changes in the areas of greatest activity for the selected sample when the excitation frequency changes. Calculations of TS distributions were carried out using Morlet wavelet (Gabor), which is characterized by the same variance in time and frequency domain [19]. It can be seen that as the frequency increases, the area of greatest activity changes. For low excitation frequencies, the distribution is mostly uniformly distributed in time. It is only the increase in the excitation frequency that causes a noticeable concentration of activity in the distribution. At the same time, as the current frequency increases, the resolution of TS distributions in time and frequency domain significantly decreases. This is particularly evident in the low band on the frequency axis $\mathrm{f}$, in the case of distributions obtained for the two highest excitation frequencies: 10 and $20 \mathrm{~Hz}$. In contrast, figure 3 shows spectrograms. In this case, as the frequency increases, a clear increase and concentration of the highest MBN activity can be observed. This is due to the acceleration of the process of reorganization of the domain structure and, as a consequence, to the superposition of individual magnetic pulses. 

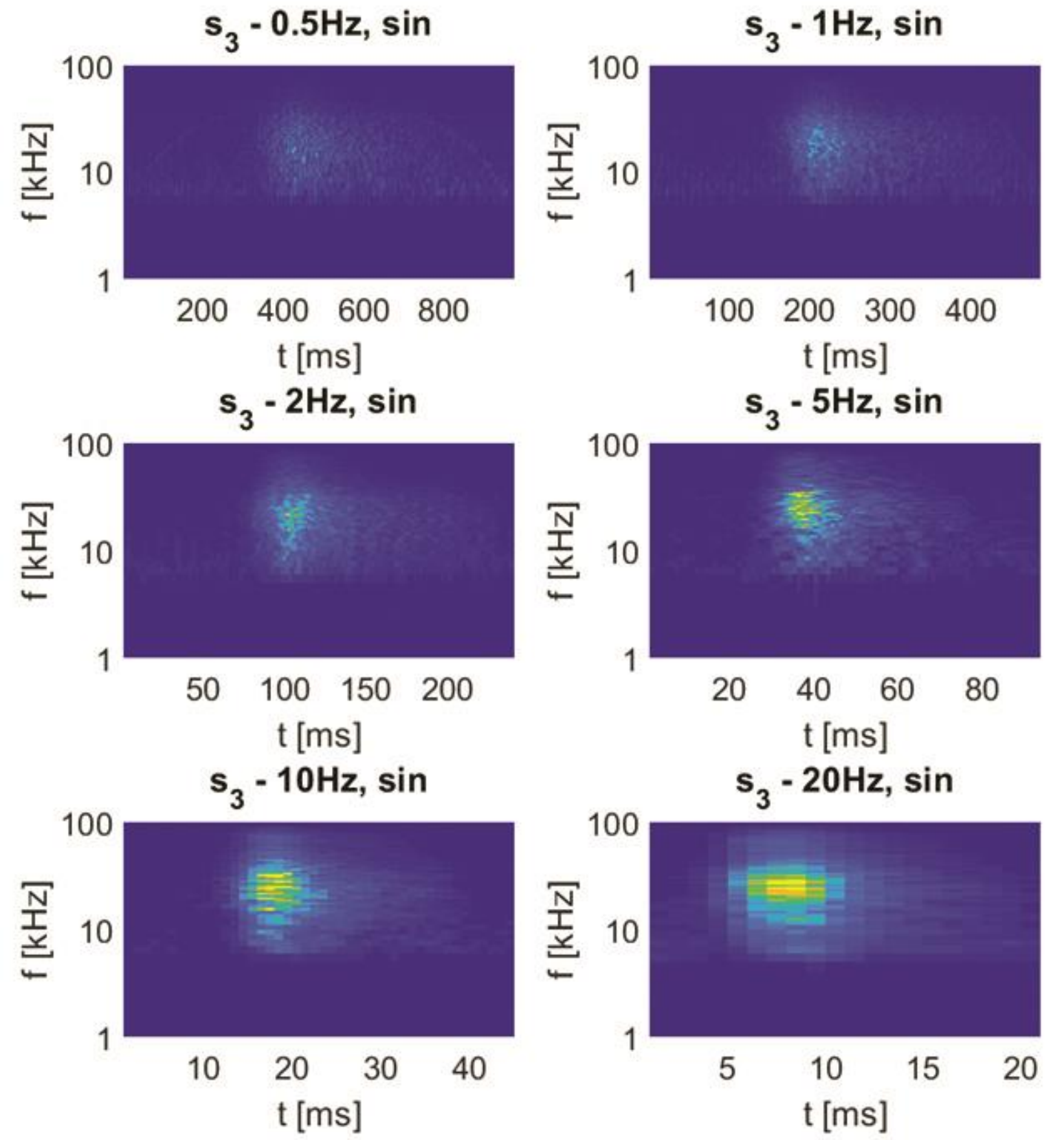

Fig. 3. Spectrograms obtained for sample s3 based on MBN signals acquired for different excitation frequency values

The next two figures (Figs. 4 and 5) present the time-frequency distributions obtained for the selected configuration of measurement parameters and various states of the tested material using both transformation methods. After comparing the corresponding distributions, a similarity can be seen in the occurrence of MBN noise activity. For sample s0, the waveforms occupy most of the bottom of the response frequency band and are stretched in time. However, in the case of $\mathrm{s}_{3}$ and s4 samples, the bandwidth increase and the shortening of the MBN noise activity time interval are clearly visible. The smallest activity can be seen for the s5 sample. Nevertheless, the distributions obtained by using the STFT transformation have a much greater visibility of the occurring changes. The presented spectrograms allow to unambiguously indicate such relationships as spectrum expansion in time, increase of the observed frequency band, increase and decrease of activity as well as shifts of the MBN noise activity center in time and frequency.

Despite the convergence of observations for both groups of results, in the case of STFT, it is possible to systematically compare noise properties in corresponding time periods and frequency ranges of distributions for different states of the tested material, as well as the values of measurement parameters used [17]. This approach is crucial in the process of non-destructive assessment of material condition and properties. Therefore, further analysis was performed only for TF distributions. 

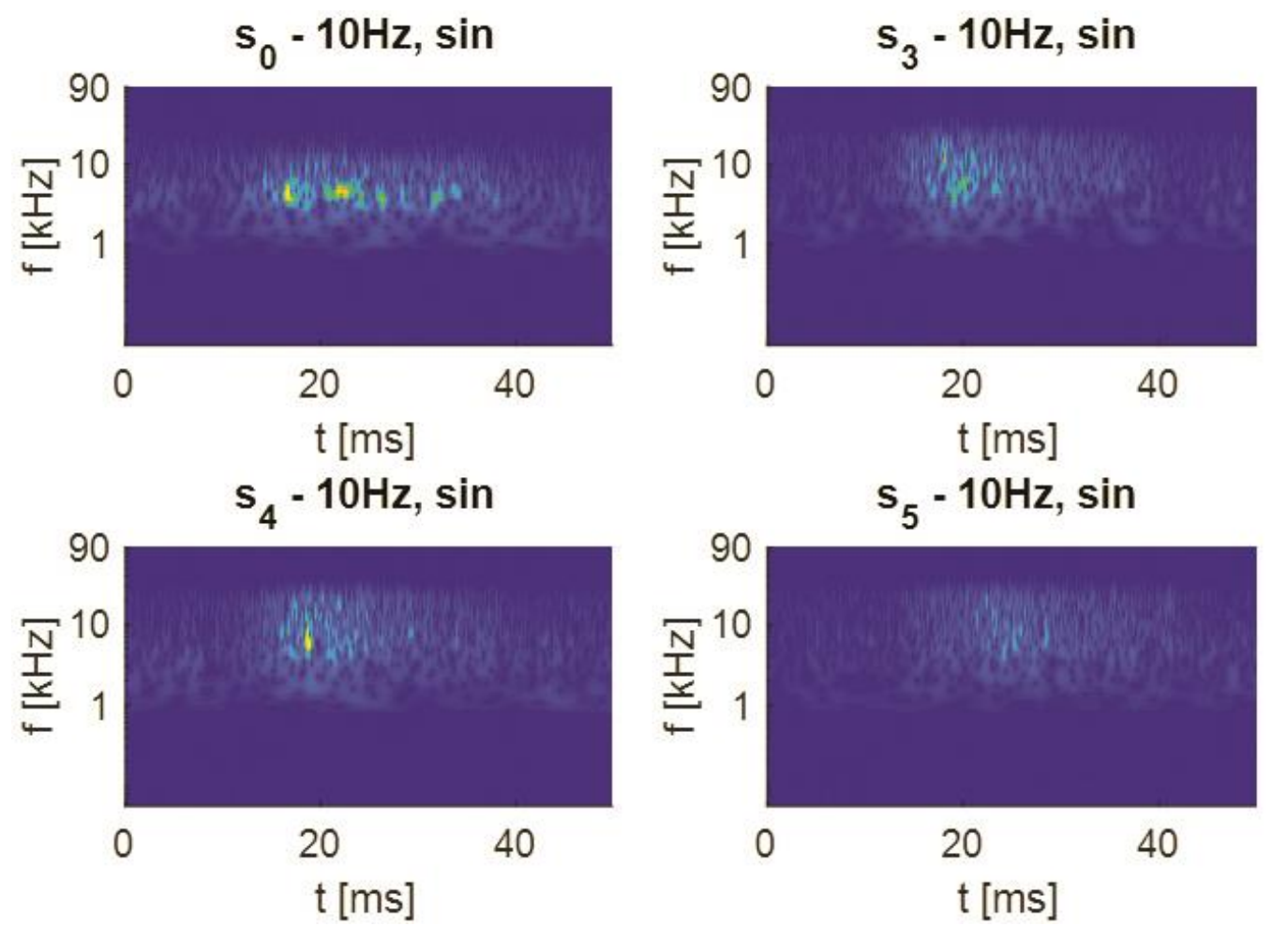

Fig. 4. Scalograms of magnetic Barkhausen noise obtained for various samples at frequency of $10 \mathrm{~Hz}$
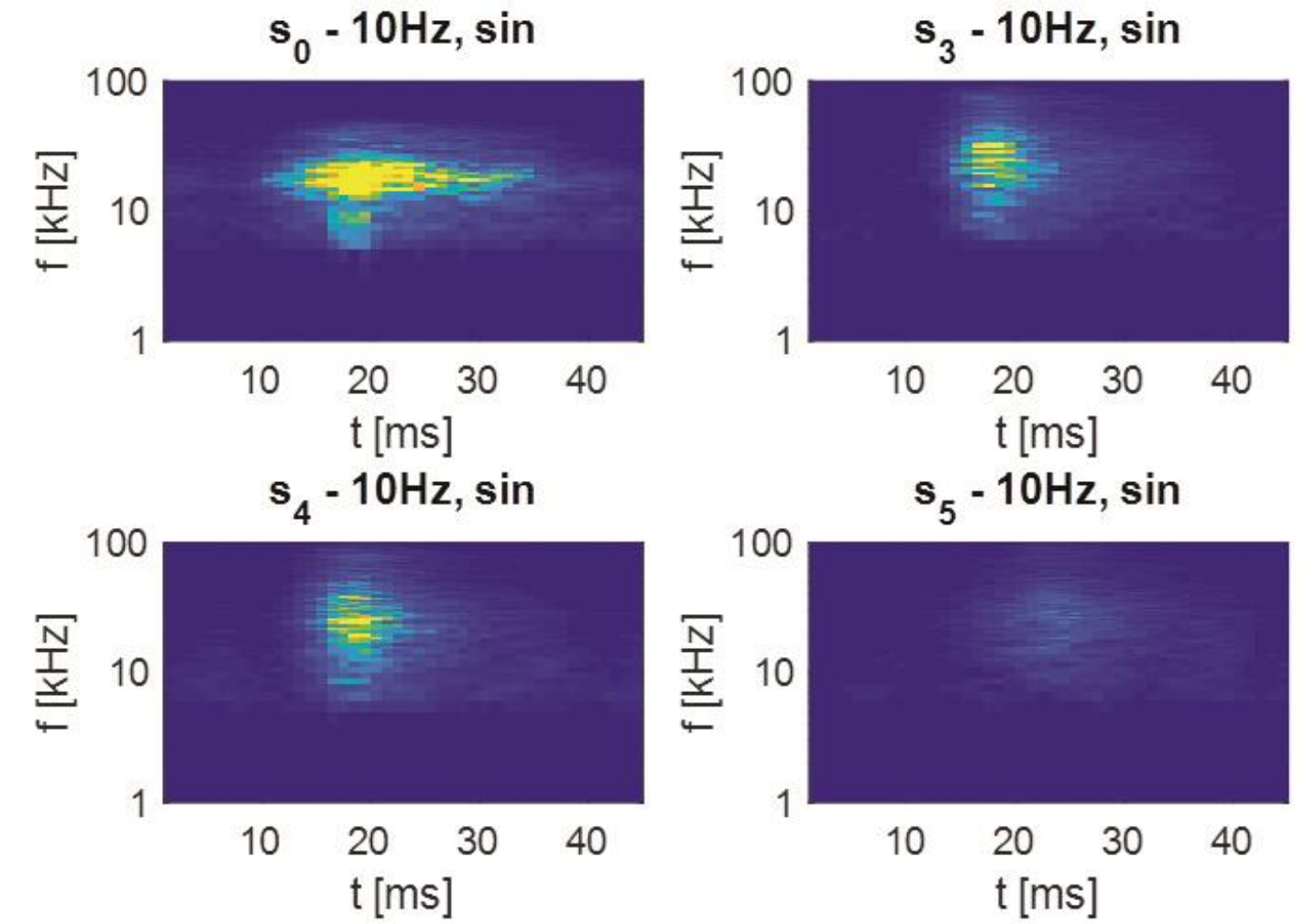

Fig. 5. Spectrograms of magnetic Barkhausen noise obtained for various samples at frequency of $10 \mathrm{~Hz}$

In the next step, after presenting the qualitative analysis and comparing the obtained scalograms and spectrograms, a quantitative analysis of the ranges of TF distribution variability was performed depending on the measurement parameters used. For the needs of this part of the analysis, a set of features was calculated, enabling, among others assessment of symmetry, center shift, flatness, homogeneity and energy concentration of TF decomposition. The set includes features such as the maximum $B N_{\text {TF____Max }}$ value,

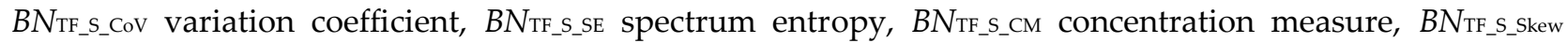
skewness or $B N_{\text {TF___S } S F}$ spectrum flatness. A detailed description of the methods used to analyze MBN signals is provided in [17]. Selected results of the analysis are presented in figures 6 and 7. 

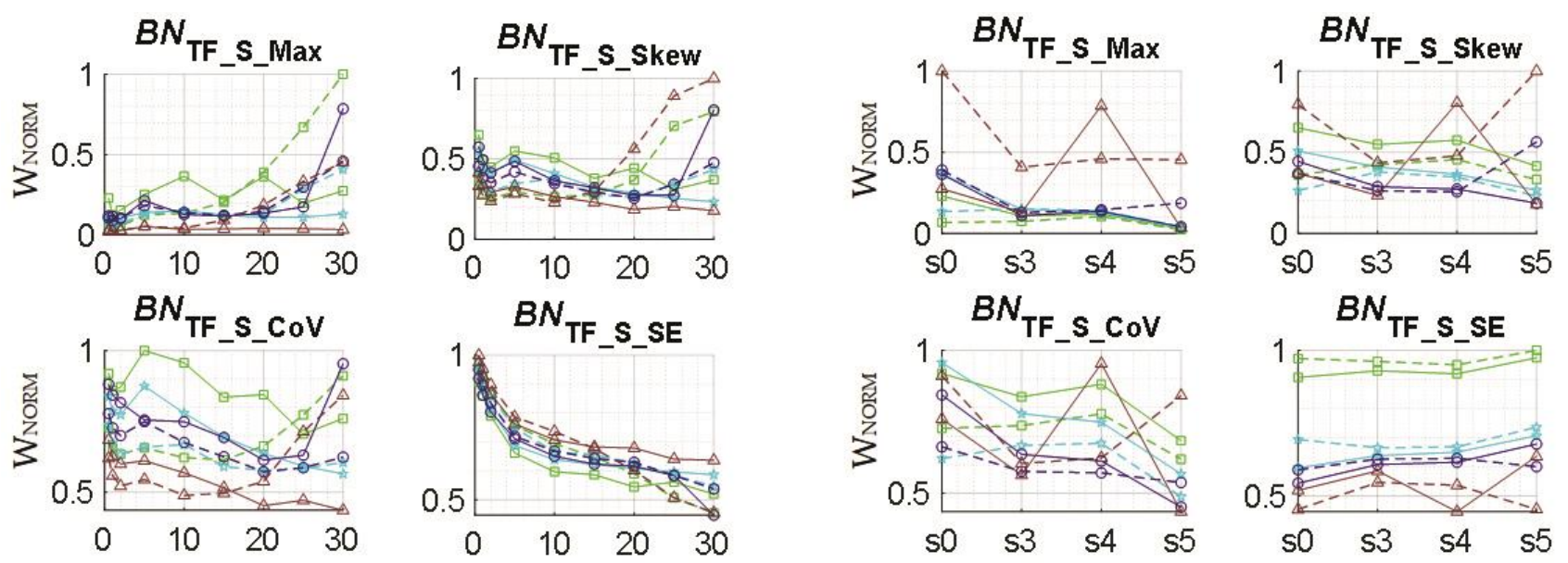

$B N_{\text {TF_S_SE }}$
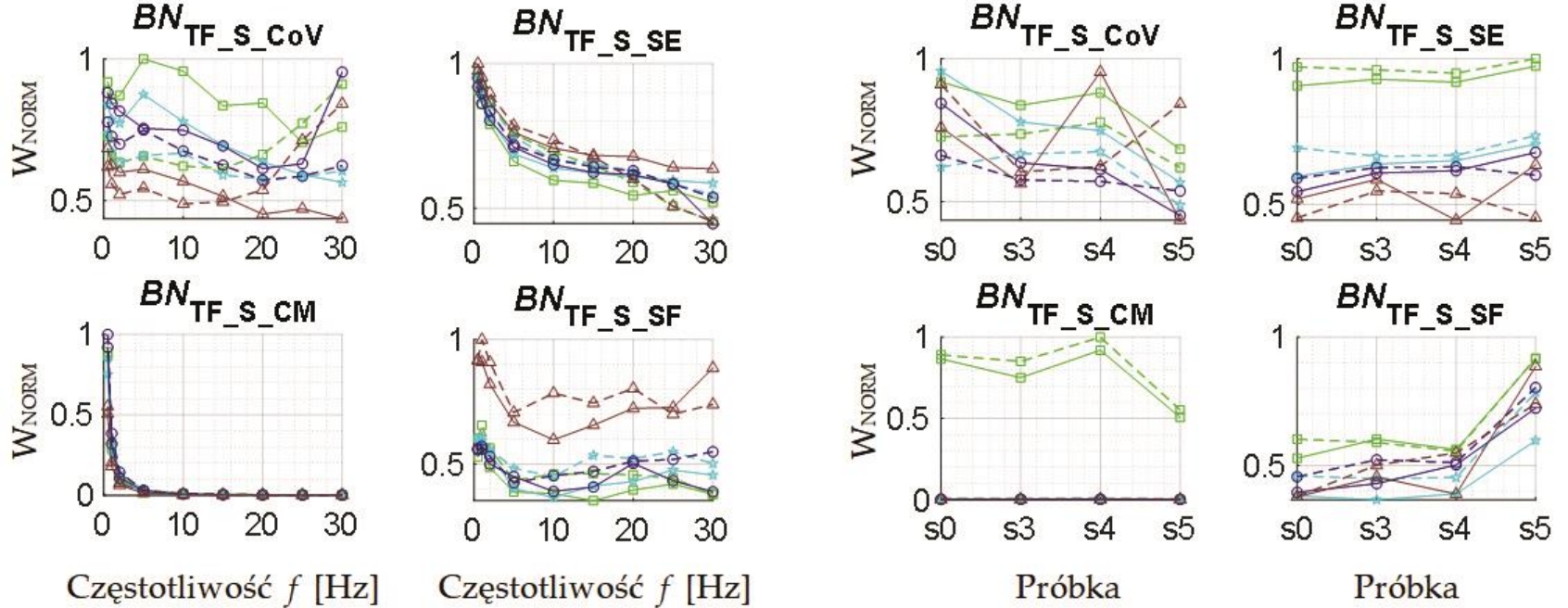

Częstotliwość $f[\mathrm{~Hz}] \quad$ Częstotliwość $f[\mathrm{~Hz}]$

Próbka

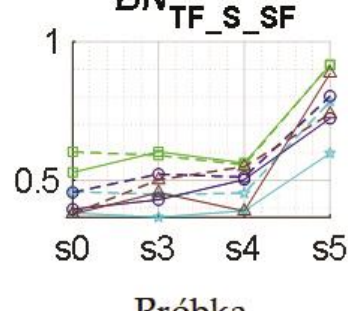

$$
\begin{aligned}
& -s_{3}, \sin -\star-s_{3}, \text { tri } \\
& \triangle s_{5}, \sin -\triangle-s_{5}, \text { tri } \\
& --s_{0}, \sin -\boxminus-s_{0} \text {, tri } \\
& -s_{4}, \sin -\ominus-s_{4}, \text { tri }
\end{aligned}
$$

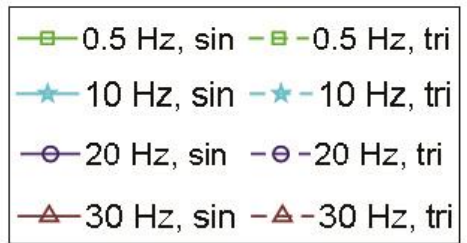

Fig. 6. Changes of parameters calculated based on the obtained spectrograms: a) as a function of the excitation frequency, b) as a function of the measurement samples

Figure 6a shows the courses of selected features of TF distributions as a function of the magnetizing current frequency, obtained for individual samples (different line color) and both shapes of the magnetizing waveforms (type of line). One can notice the convergence of trends obtained for various samples, while the size of the increases observed for higher excitation frequency values depends on the shape of its course. The largest difference in value levels between waveforms obtained for individual

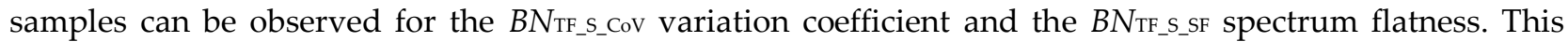
allows greater discrimination of states (changes in properties) of the tested material. In the case of $B N_{T F} S_{-} \mathrm{Cov}$, the waveform values first increase in the frequency range up to $10 \mathrm{~Hz}$, then decrease to about 25 $\mathrm{Hz}$ and begin to increase again for the most part. However, in the case of $B N_{\mathrm{TF} \mathrm{S}_{-} \mathrm{SF}}$ waveforms, the local minimum waveforms for a frequency of about $10 \mathrm{~Hz}$ are noticeable. On the other hand, the smallest variability was obtained in the case of the BNTF___cм concentration measure. For this feature, similar waveforms were obtained for all tested samples. Only in the low frequency range of the magnetizing current (up to $10 \mathrm{~Hz}$ ), small differences between the waveforms recorded for individual samples can be seen. In Figure $6 \mathrm{~b}$, the obtained parameter distributions are presented as a function of the steel samples tested, which allows observation of changes in levels of individual features depending on the sample tested. It can again be seen that the $B N_{T T F_{-} S_{-} C_{0}}$ and $B N_{\text {TF____SF }}$ parameters are characterized by high variability. In addition, the $B N_{\text {TF____CM }}$ parameter guarantees clear discrimination in the low frequency range. Despite the visible differences, unambiguous assessment of the quality of information of individual features is not easy. Therefore, to determine the variability of individual features depending on the frequency and type of excitation course, the cumulative values of their standard deviation were calculated. The results of this operation for selected features are shown in figure 7. Three groups can be distinguished from the set of analyzed features. The first group are parameters that have a higher cumulative standard deviation for higher frequencies, e.g. BNTF___Max. Another group of parameters are those that are characterized by increased discrimination in the low frequency range, e.g. BNTF___см. The last group consists of parameters that have a fairly balanced discrimination in a wide frequency range, e.g. $B N_{\text {TF___SF. }}$ 

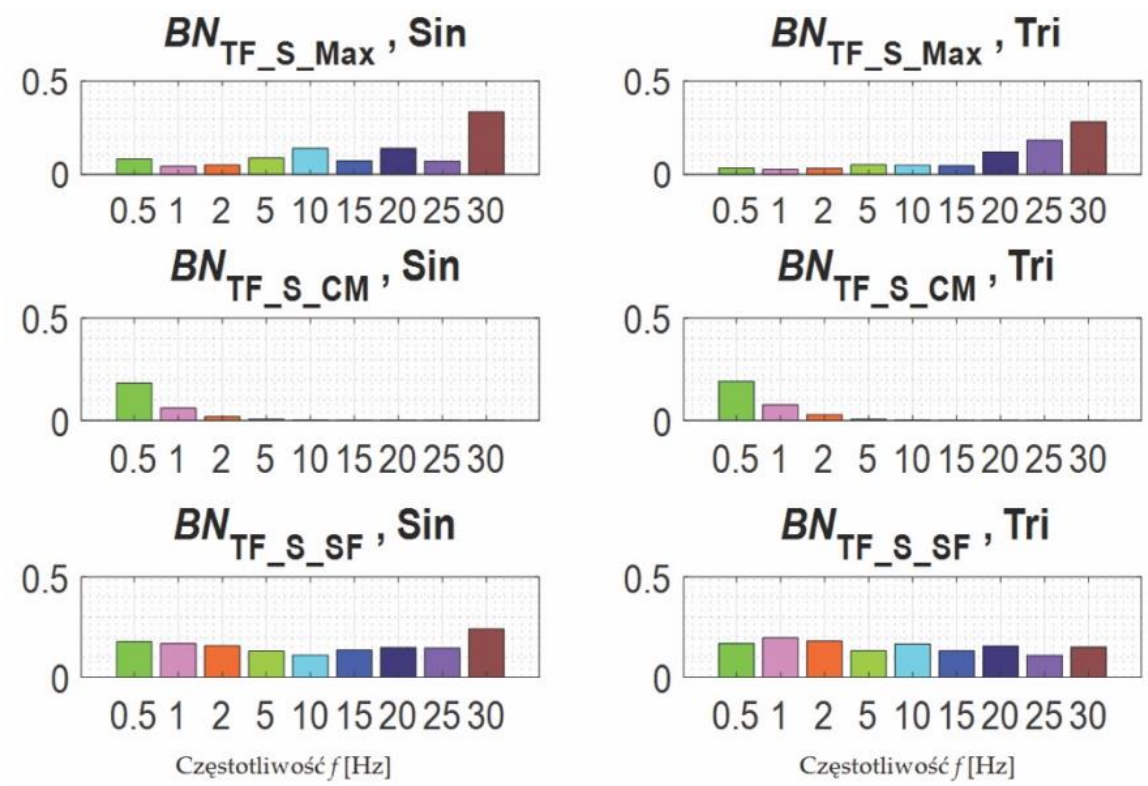

Fig. 7. Calculated values of cumulative standard deviation for selected parameters

\section{Summary}

Measurement conditions testing is a key element in the measurement system analysis process. Choosing the right measured values increases the efficiency of using the system. As a result, it is also possible to develop appropriate algorithms to shorten the analysis and data mining process. Despite the visible ranges of higher discrimination of individual parameters, it is not possible to determine clearly dominant one selected parameter that will guarantee high detection efficiency for different states of the tested material. The results obtained justify the need to build multi-parameter rules based on data obtained in a wide spectrum of variability of measurement conditions. Consequently, this may allow for higher efficiency of the measuring system.

Author Contributions: writing M.M., validation G.P., formal analysis M.M. and G.P., resources M.M., methodology G.P., conceptualization M.M. and G.P., software M.M. and G.P.

Conflicts of Interest: The authors declare no conflict of interest.

\section{References}

[1] Makowska K., Kowalewski Z.L., Augustyniak B., Piotrowski L., Determination of mechanical properties of P91 steel by means of magnetic Barkhausen emission, Journal of Theoretical and Applied Mechanics, 2014, Vol. 52(1), 181-188. [CrossRef]

[2] Kleber X., Vincent A., On the role of residual internal stresses and dislocations on Barkhausen noise in plastically deformed steel, NDT \& E International, 2004, Vol. 37(6), 439-445. [CrossRef]

[3] Psuj G., Fusion of Multiple Parameters of Magnetic Testing Results for Damage Assessment of Loaded Steel Structures, Studies in Applied Electromagnetics and Mechanics, 2015, Vol. 40, 192-199. [CrossRef]

[4] Szymanik B., Psuj G., Infrared and electromagnetic inspection of steel structures under load, Quantitative InfraRed Thermography Journal, 2016, Vol. 13, 232-241. [CrossRef]

[5] Deng Y., Li Z., Chen J., Qi X., The effects of the structure characteristics on Magnetic Barkhausen noise in commercial steels, Journal of Magnetism and Magnetic Materials, 2018, Vol. 451, 276-282. [CrossRef]

[6] Bartošová I., Veterníková J., Slugeň V., Study of candidate materials for new reactor systems using positron annihilation spectroscopy and Barkhausen noise, Nuclear Engineering and Design, 2014, Vol. 273, 376-380. [CrossRef]

[7] Yamazaki T., Furuya Y., Nakao W., Experimental evaluation of domain wall dynamics by Bark-hausen noise analysis in Fe30Co70 magnetostrictive alloy wire, Journal of Magnetism and Magnetic Materials, 2019, Vol. 475, 240-248. [CrossRef]

[8] Ding S., Tian G., Dobmann G., Wang P., Analysis of domain wall dynamics based on skewness of magnetic Barkhausen noise for applied stress determination, Journal of Magnetism and Magnetic, 2017, Vol. 421, $225-229$.

[CrossRef] 
[9] Pérez-Benitez J.A., Padovese L.R., Capó-Sánchez J., Anglada-Rivera J., Investigation of the magnetic Barkhausen noise using elementary signals parameters in 1000 commercial steel, Journal of Magnetism and Magnetic, 2003, Vol. 263, 72-77. [CrossRef]

[10] Psuj G., Maciusowicz M., Chudzik P., Influence of measurement conditions on the Magnetic Bark-hausen Noise properties, In Proceedings of the 2018 International Interdisciplinary PhD Workshop (IIPhDW), 2018, 326-329. [CrossRef]

[11] Kwaśniewski J., Roskosz M., Witoś M., Molski S., Applications of Magnetometric Sensors Based on Amorphous Materials in Diagnostics of Wire Ropes, Archives of Mining Sciences, 2018, Vol. 63(1), 221-227. [Hyperlink]

[12] Sorsa A., Santa-aho S., Vippola, M., Lepistö T., Leiviskä K., Utilization of frequency-domain information of Barkhausen noise signal in quantitative prediction of material properties, AIP Conf. Proc., 2014, Vol. 1581(1), 1256-1263. [CrossRef]

[13] Kypris O., Nlebedim I.C., Jiles D.C., A model for the Barkhausen frequency spectrum as a function of applied stress, Journal of Applied Physics, 2014, Vol. 115(8), 083906. [CrossRef]

[14] Tomkowski R., Jonsson S., Lundin P., Nerman P., Penetration depth investigation of Barkhausen noise signal for case-hardened components, Dresden, Germany, 2017.

[15] Psuj G., Maciusowicz M., Analysis of time-frequency representation of Magnetic Barkhausen noise for the need of damage evaluation of steels elements, In Proceedings of the 2018 International Interdisciplinary PhD Workshop (IIPhDW), 2018, pp. 108-110. [CrossRef]

[16] Padovese L., Martin N., Millioz F., Time-frequency and Time-Scale analysis of Barkhausen noise signals, Proceedings of the Institution of Mechanical Engineers, Part G: Journal of Aerospace Engineering, 2009, Vol. 223(5), 577588. [CrossRef]

[17] Maciusowicz M., Psuj G., Use of Time-Dependent Multispectral Representation of Magnetic Barkhausen Noise Signals for the Needs of Non-Destructive Evaluation of Steel Materials, Sensors, 2019, Vol. 19(6), 1443. [CrossRef]

[18] Psuj G., Maciusowicz M., The use of time-dependent spectral representation of Barkhausen noise signal for the needs of non-destructive evaluation of steel elements, Badania Nieniszczace i Diagnostyka, 2018, 69-71. [CrossRef]

[19] Boashash B., Ouelha S., Designing high-resolution time-frequency and time-scale distributions for the analysis and classification of non-stationary signals: a tutorial review with a comparison of features performance, Digital Signal Processing, 2018, Vol. 77, 120-152. [CrossRef]

[20] Łopato P., Psuj G., Herbko M., Maciusowicz M., Evaluation of stress in steel structures using electromagnetic methods based on utilization of microstrip antenna sensor and monitoring of AC mag-netization process, Informatyka Automomatyka Pomiary W Gospododarce i Ochronie Środowiska, 2016, nr 4. [CrossRef]

(C) 2019 by the authors. Submitted for possible open access publication under the terms and conditions of the Creative Commons Attribution (CC BY) license (http://creativecommons.org/licenses/by/4.0/). 